FRUET et all, v(11), no 11, p. 2307-2312, JUN, 2013.

Rev. Elet. em Gestão, Educação e Tecnologia Ambiental (e-ISSN: 2236-1170)

\title{
PERDAS ECONÔMICAS POR CONDENAÇÃO DE ÓRGÃOS SUÍNOS EM MATADOUROS SOB SERVIÇO DE INSPEÇÃO MUNICIPAL
}

\section{ECONOMIC LOSSES DUE TO CONDEMNATION OF SWINE ORGANS IN SLAUGHTERHOUSES UNDER MUNICIPAL INSPECTION SERVICE}

\author{
Ana Paula Burin Fruet ${ }^{1}$, Análie Scortegagna ${ }^{2}$, Edom de Avila Fabricio ${ }^{3}$, Jackeline Karsten Kirinus ${ }^{4}$, \\ Andréa Cristina Dörr ${ }^{5}$, José Laerte Nörnberg ${ }^{6}$ \\ ${ }^{1}$ Universidade Federal de Santa Maria (UFSM) - Aluna de mestrado do Programa de Pós-graduação em Ciência e Tecnologia de \\ Alimentos. E-mail: ap_burin@hotmail.com \\ ${ }^{2}$ Médica Veterinária: analiescortegagna@gmail.com \\ ${ }^{3}$ UFSM - Aluno do curso de Zootecnina: edomfabricio@hotmail.com \\ ${ }^{4}$ UFSM - Aluna de doutorado do Programa de Pós-graduação em Ciência e Tecnologia de Alimentos: jackeline.kirinus@gmail.com \\ ${ }^{5}$ UFSM - Profa. Adjunto do Departamento de Ciências Econômicas: andreadoerr@yahoo.com.br \\ ${ }^{6}$ UFSM - Prof. Associado do Departamento de Tecnologia e Ciência dos Alimentos: jlnornberg@gmail.com
}

http://dx.doi.org/10.5902/223611708727

\begin{abstract}
RESUMO
O objetivo deste trabalho foi mensurar as perdas causadas pela condenação de órgãos suínos em matadouros sob fiscalização do serviço de inspeção municipal (SIM) em Santa Maria, RS durante 12 meses consecutivos. O estudo foi realizado através do levantamento de dados de matadouros do município de Santa Maria, Rio Grande do Sul, Brasil, junto ao SIM. Os dados registrados foram o número de animais abatidos, número de órgãos condenados e principais lesões que levaram as condenações, no período de outubro de 2010 a setembro de 2011. Durante o período de avaliação, 6.193 animais foram abatidos. Observou-se que 5.511 órgãos foram condenados, com destaque as condenações por lesões de aspiração de sangue $(n=1.231)$, migração larval $(n=991)$ e cisto renal $(n=807)$. A pesquisa revelou que anualmente $\mathrm{R} \$ 11.955,66$ é desperdiçado com condenação de órgãos. A migração larval no

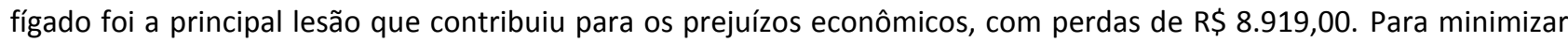
as perdas econômicas é necessário agir nos pontos críticos de condenação, o principal ponto observado no estudo foi o descarte de fígado por migração larval.
\end{abstract}

Palavras-chave: fígado, subprodutos, lesões, migração, larval, prejuízo financeiro

\begin{abstract}
This study aimed to measure the economic losses caused by the condemnation of swine organs in municipal slaughterhouses under the supervision of the municipal inspection system (MIS) from the city of Santa Maria-RS during 12 months in a row. The study was conducted by collecting data from slaughterhouses in Santa Maria, Rio Grande do Sul, Brazil, in MIS. The registered data took into account the number of animals slaughtered, number of condemned organs and major lesions that led to condemnation from October 2010 to September 2011. During the evaluation period, 6,193 animals were slaughtered. It was found that 5,511 organs were condemned, mostly because of aspiration of blood $(n=1,231)$, larval migration in liver $(n=991)$, and renal cyst $(n=807)$. The survey revealed that $\mathrm{R} \$ 11,955.66$ is wasted annually in condemnation of organs. It is noteworthy that the main injury that contributed to the economic damage was larval migration in liver, which discard amount reached $R \$ 8,919.00$. In order to reduce economic losses, it is necessary to do something against the critical points of condemnation, in which the main one observed in the study was the disposal of liver caused by larval migration.
\end{abstract}

Keywords: liver, by-products, lesions, larval migration, economic losses 


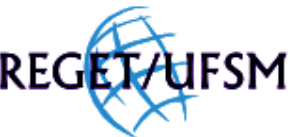

FRUET et all, v(11), no 11, p. 2307-2312, JUN, 2013.

Rev. Elet. em Gestão, Educação e Tecnologia Ambiental (e-ISSN: 2236-1170)

\section{INTRODUÇÃO}

Na cadeia produtiva de carne os órgãos são subprodutos importantes economicamente, pois agregam valor à produção de matadouros por serem potenciais fontes de ingredientes alimentares para a população mundial. Portanto, é provável que os subprodutos alimentares tenham uma maior relevância no futuro (CHIBA, 2005; KALE et al., 2011).

No momento da inspeção post mortem todos os órgãos e tecidos são examinados e observadas suas características externas, estes também passam por palpação e cortes sobre o parênquima (BRASIL, 1997). Nesse processo de inspeção sanitária, quando os órgãos e carcaças apresentam alterações, são condenados e constituem um prejuízo econômico direto para a indústria frigorífica (SOUZA et al., 2007; KALE et al., 2011).

As principais alterações descritas na literatura são as lesões causadas por migração de parasitas (LIGNON et al., 1985; PFUETZENREITER \& ÁVILA-PIRES, 2000; BEM, 2008; AGUIAR, 2009). No entanto, as perdas econômicas geradas por tais condenações são pouco relatadas.

Dessa forma, os objetivos deste trabalho foram mensurar as perdas em matadouros municipais de Santa Maria, RS, causadas pela condenação de órgãos suínos através da quantificação do número de órgãos condenados; identificar as principais lesões que levaram as condenações e estimar perdas econômicas para indústria com o descarte dos subprodutos.

\section{METODOLOGIA}

Essa é uma pesquisa quantitativa que utilizou como instrumento de análise o levantamento de dados primários e secundários (BARBETTA, 2011) da população total de suínos abatidos em matadouros do município de Santa Maria, Rio Grande do Sul, sob fiscalização do Serviço de Inspeção Municipal (SIM). Os dados secundários foram coletados pelos médicos veterinários e agentes do SIM, no período de outubro de 2010 a março de 2011. E por sua vez, os dados primários, foram coletados pelos autores em visitas de rotina dos médicos veterinários do SIM no mesmo município entre abril e setembro de 2011.

A coleta de dados totalizou o período de um ano para que os resultados não sofressem interferência de possíveis sazonalidades de lesões. Durante o período de avaliação, 6.193 suínos foram abatidos, dos quais identificou-se o número de órgãos condenados e lesões encontradas.

Os dados foram tabulados, posteriormente realizados os cálculos de prevalência das condenações e prejuízos econômicos. Para essa finalidade, foram utilizados os pesos dos órgãos de acordo com Thornton (1969) e valores de venda dos subprodutos estipulados pelos matadouros acompanhados no município, cujo preço é de $\mathrm{R} \$ 3,00$ por quilograma de órgão.

\section{RESULTADOS E DISCUSSÃO}

Essa pesquisa teve como base a investigação de três aspectos: número de órgãos condenados, as principais lesões que causaram o descarte dos órgãos e os prejuízos decorrentes. Com essa finalidade foram inspecionados 43.351 órgãos (Tabela 1). O rendimento anual de cada 


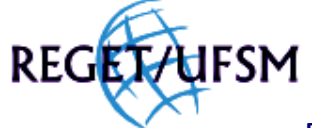

FRUET et all, v(11), no 11, p. 2307-2312, JUN, 2013.

Rev. Elet. em Gestão, Educação e Tecnologia Ambiental (e-ISSN: 2236-1170)

subproduto avaliado está relatado na tabela 1 . Os dados revelam que o fígado contribui com 53,08\% para o rendimento financeiro dos órgãos em matadouros do município de Santa Maria, RS.

Tabela 1. Demonstrativo do número de órgãos inspecionados, peso (Kg), rendimento financeiras de cada órgão anualmente

\begin{tabular}{lrrrrrrr}
\hline \multicolumn{1}{c}{ Órgão } & Cabeça & Língua & Fígado & Pulmão & Rins & Coração & Total \\
\hline $\begin{array}{l}\text { Número órgãos } \\
\text { inspecionados }\end{array}$ & 6.193 & 6.193 & 6.193 & 6.193 & 12.386 & 6.193 & 43.351 \\
& & & & & & & \\
& & & & & & & \\
$\begin{array}{l}\text { Peso dos órgãos } \\
\text { inspecionados (Kg) }\end{array}$ & 12.386 & $1.238,6$ & 18.579 & 7 & 6 & $1.238,6$ & 6 \\
& & & & & & & \\
Rendimento anual & $37.158,0$ & & $55.737,0$ & & & \\
em Reais (R\$) & 0 & $3.715,80$ & 0 & - & $4.681,91$ & $3.715,80$ & $105.008,5$ \\
\hline
\end{tabular}

Fonte: Dados da pesquisa elaborada pelos autores.

O número de órgãos condenados foi de 5.511 (Tabela 2), o que representa uma perda de $12,71 \%$ de subprodutos. Observou-se que o principal órgão condenado foi o pulmão seguido do rim, fígado, coração, cabeça e a língua.

As condenações de pulmões ( $n=2.276$ ) assim como de rins ( $n=1.670)$, embora numericamente importantes, são pouco relevantes no âmbito de prejuízos financeiros. Este fato é decorrente do baixo peso renal e pelo pulmão não apresentar valor comercial para as indústrias avaliadas.

A lesão de maior prevalência foi causada por aspiração de sangue (Tabela 2), o achado pulmonar citado, assim como enfisema pulmonar, podem estar associados a doenças, porém com freqüência são causados por ineficiência de tecnologia de abate durante o procedimento de abate (GREGORY et al., 2009; MELLAU et al., 2010). Os dados descritos por D'Alencar et al. (2011) demonstraram semelhança aos encontrados neste estudo, no entanto a principal lesão pulmonar relatada foi pneumonia. Esta é a segunda lesão encontrada nos frigoríficos analisados.

Apesar da lesão de cisticercose não ser relevante no estudo, é uma doença parasitária considerada zoonose e apresenta grande importância à saúde pública (PFUETZENREITER \& ÁVILAPIRES, 2000; SANTOS et al., 2008; KHANIKI, 2010; SANTOS et al., 2010). Nos suínos, o desenvolvimento completo dos cisticercos se dá entre 60 e 75 dias após a infecção e a larva permanece viável para parasitar o homem durante anos (SILVA, 2004). A intensificação da produção de suínos aliado ao tempo de desenvolvimento dos cisticercos podem ser uma explicação para o baixo número de achados de cisticercose.

O valor da renda bruta que seria arrecadada pelos matadouros do município de Santa Maria, RS com as vendas de órgãos suínos é de R\$105.008,51 (Tabela 1). No entanto, a pesquisa revelou que $11,39 \%$ desta renda é perdida no momento da inspeção post mortem dos órgãos. Este montante equivale a uma perda de $\mathrm{R} \$ 11.955,66$ na renda anual de produção. Dentre todos os órgãos analisados, ficou evidente a maior participação do fígado nas perdas por condenação 
Rev. Elet. em Gestão, Educação e Tecnologia Ambiental (e-ISSN: 2236-1170)

(Tabela 2). O órgão foi o responsável pelo prejuízo de $\mathrm{R} \$ 10.809,00$, o equivalente a 90,41\% do valor total desperdiçado.

Tabela 2. Números de órgãos condenados, assim como, o valor (R\$) e percentagem (\%) desperdiçados por condenações durante um ano

\begin{tabular}{|c|c|c|c|c|c|}
\hline \multirow[t]{2}{*}{ Órgãos } & \multirow[t]{2}{*}{ Lesões } & \multicolumn{2}{|c|}{ Número de condenados } & \multicolumn{2}{|c|}{$\begin{array}{c}\text { Total de condenação em } \\
\text { Reais }\end{array}$} \\
\hline & & $\mathrm{N}^{\circ}$ & $\%$ & $\mathrm{~N}^{\circ}$ & $\%$ \\
\hline \multicolumn{6}{|l|}{ Pulmão } \\
\hline & Aspiração de sangue & 1.231 & 22,34 & 0,00 & 0,00 \\
\hline & Pneumonia & 399 & 7,24 & 0,00 & 0,00 \\
\hline & Enfizema pulmonar & 279 & 5,06 & 0,00 & 0,00 \\
\hline & Outros & 367 & 6,66 & 0,00 & 0,00 \\
\hline & Subtotal & 2.276 & 41,30 & 0,00 & 0,00 \\
\hline \multicolumn{6}{|l|}{ Rim } \\
\hline & Cisto renal & 807 & 14,64 & 305,05 & 2,55 \\
\hline & Nefrite & 291 & 5,28 & 110,00 & 0,92 \\
\hline & Isquemia & 282 & 5,12 & 106,60 & 0,89 \\
\hline & Outros & 290 & 5,26 & 109,62 & 0,92 \\
\hline & Subtotal & 1.670 & 30,30 & 631,26 & 5,28 \\
\hline \multicolumn{6}{|l|}{ Fígado } \\
\hline & Migração larval & 991 & 17,98 & $8.919,00$ & 74,60 \\
\hline & Aderência & 92 & 1,67 & 828,00 & 6,93 \\
\hline & Contaminação & 37 & 0,67 & 333,00 & 2,79 \\
\hline & Outros & 81 & 1,47 & 729,00 & 6,10 \\
\hline & Subtotal & 1.201 & 21,79 & $10.809,00$ & 90,41 \\
\hline \multicolumn{6}{|l|}{ Coração } \\
\hline & Aderência & 206 & 3,74 & 123,60 & 1,03 \\
\hline & Pericardite & 72 & 1,31 & 43,20 & 0,36 \\
\hline & Contaminação & 28 & 0,51 & 16,80 & 0,14 \\
\hline & Outros & 02 & 0,04 & 1,20 & 0,01 \\
\hline & Subtotal & 308 & 5,59 & 184,80 & 1,55 \\
\hline \multicolumn{6}{|l|}{ Cabeça } \\
\hline & Rinite atrófica & 44 & 0,80 & 264,00 & 2,21 \\
\hline & Contaminação & 10 & 0,18 & 60,00 & 0,50 \\
\hline & Cisticercose & 01 & 0,02 & 6,00 & 0,05 \\
\hline & Subtotal & 55 & 1,00 & 330,00 & 2,76 \\
\hline \multicolumn{6}{|l|}{ Língua } \\
\hline & Abscesso & 01 & 0,02 & 0,60 & 0,01 \\
\hline & Subtotal & 01 & 0,02 & 0,60 & 0,01 \\
\hline & Total & 5.511 & 100 & $11.955,66$ & 100 \\
\hline
\end{tabular}

Fonte: Dados da pesquisa elaborada pelos autores. 


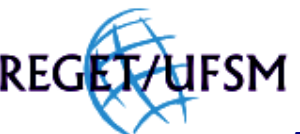

FRUET et all, v(11), no 11, p. 2307-2312, JUN, 2013.

Rev. Elet. em Gestão, Educação e Tecnologia Ambiental (e-ISSN: 2236-1170)

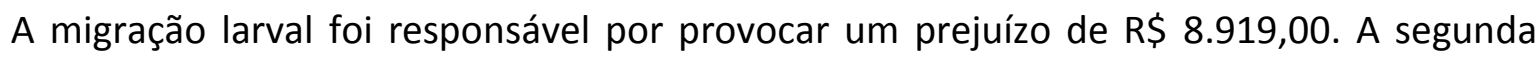
principal causa de perdas financeiras dentro das lesões hepáticas e nas lesões de todos os órgãos analisados foi aderência. Esta lesão representou uma perda de $R \$ 828,00$, o equivalente a $6,93 \%$ do prejuízo final.

Devido aos órgãos serem subprodutos que agregam valor na renda dos frigoríficos tornase indispensável minimizar as perdas por condenação (CHIBA, 2005; KALE et al., 2011). As condenações referidas apresentam perdas econômicas diretas para a indústria (SOUZA et al., 2007; KALE et al., 2011) e indiretas para o produtor, pois animais com órgãos comprometidos não terão o mesmo desempenho de produção e econômico quando comparados com animais hígidos (PEREIRA et al., 2006; MAS-COMA et al., 2009; DUTRA et al., 2010).

Dessa forma, como o fígado representou o maior prejuízo econômico neste estudo, é necessário focar as atenções para evitar condenações e diminuir perdas desse órgão. Logo, como a principal condenação apontada no levantamento foi provocada por migração larval, fazer uso do manejo terapêutico para combater parasitas de suínos pode minizar os achados. O principal parasito responsável por causar lesões correspondente a manchas claras no fígado e, por consequencia, a condenação do órgão é o Ascaris suum (AGUIAR, 2009). Entre as medidas profiláticas, um plano de ação que pode ser elaborado e executado pela indústria é o estímulo aos produtores para avaliar o relatório de abate e estimar a saúde do plantel.

\section{CONCLUSÕES}

Foram condenados números expressivos de órgãos de suínos. As lesões de maior prevalência foram causadas por aspiração de sangue, migração larval no fígado e cisto renal respectivamente. As perdas por condenação de órgãos em matadouros sob inspeção municipal de Santa Maria, RS são de R\$ 11.955,66 e a lesão hepática causada por migração larval foi a responsável pela maior parte das perdas econômicas.

\section{AGRADECIMENTOS}

À secretaria de desenvolvimento rural de Santa Maria, RS, por disponibilizar os dados desta pesquisa, e em especial ao médico veterinário Alexandre Caetano por estimular o estudo, pelo auxílio prestado e por acreditar na importância dos resultados.

\section{REFERÊNCIAS BIBLIOGRÁFICAS}

AGUIAR, P. C. Aspectos epidemiológicos das parasitoses gastrointestinais de suínos naturalizados de criações familiares do Distrito Federal. Brasília: UnB, 2009. Dissertação (Mestrado em Saúde animal), Universidade de Brasília, 2009.

BARBETTA, P. A. Estatística aplicada às Ciências Sociais. 7.ed. Florianópolis: Ed. da UFSC, 2011. 315p.

BEM, E. L. D. Epidemiologia e anatomopatologia de lesões pulmonares de suínos em um matadouro em cascavel PR. Curitiba: UFPR, 2008. Dissertação (Mestrado em Ciência Veterinária), Universidade Federal do Paraná, 2008. 


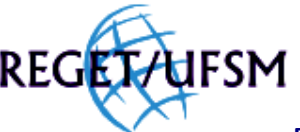

FRUET et all, v(11), no 11, p. 2307-2312, JUN, 2013.

Rev. Elet. em Gestão, Educação e Tecnologia Ambiental (e-ISSN: 2236-1170)

BRASIL. Decreto 20.691-52. Regulamento de Inspeção Industrial e Sanitária de Produtos de Origem Animal (RIISPOA). Brasília: Ministério da Agricultura, Pecuária e Abastecimento, 1997. Diário Oficial da União de 07 de jul.1952, página 10.785. Brasília/DF, 1997.

CHIBA, L. I. By-product feeds: animal origin. In: Encyclopedia of Animal Science. 2. ed. Alabama: Taylor \& Francis, 2005. p. 169-174.

D’ ALENCAR, A. S. et al. Manejo higiênico-sanitário e lesões pulmonares em suínos na Região Metropolitana de Recife e Zona da Mata de Pernambuco, Brasil. Semina: Ciências Agrárias, v. 32, n. 3, p. 1111-1122, 2011.

DUTRA, L. H. et al. Mapping risk of bovine fasciolosis in the south of Brazil using geographic information systems.Veterinary Parasitology, v. 169, p.76-81, 2010.

GREGORY, N. G.; WENZLOWOWICZ, M. V; HOLLEBEN, K. V. Blood in the respiratory tract during slaughter with and without slunning in cattle. Meat science, v. 82, n. 1, p. 13-16, 2009.

KALE, M. C. et al. Determination of by-product economic values for slaughtered cattle and sheep. Kafkas Universitesi Veteriner Fakultesi Dergisi, v. 17, n. 4, p. 551-556, 2011.

KHANIKI, Gh. R. J. et al. Prevalence of bovine cysticercosis in slaughtered cattle in Iran. Tropical Animal Health and Production, v. 42, p. 141-143, 2010.

LIGNON, G. B. et al. Ação do Oxibendazole frente à migração de larvas de Ascaris suum em suínos. Comunicado técnico, EMBRAPA, 1985.

MAS-COMA, S.; VALERO, M. A.; BARGUES, M. D. Climate change effects on trematodiases, with emphasis on zoonotic fascioliasis and schistosomiasis. Veterinary Parasitology, v. 163, p. 264-280, 2009.

MELLAU, L. S. B.; NONGA, H. E.; KARIMURIBO, E. D. A slaughterhouse survey of lung lesions in slaughtered stocks at Arusha, Tanzania. Preventive Veterinary Medicine, v. 97, p. 77-82, 2010.

PEREIRA, M. A. V. da C.; SCHWANZ, V. S.; BARBOSA, C. G. Prevalência da cisticercose em carcaças de bovinos abatidos em matadouros-frigoríficos do estado do Rio de Janeiro, submetidos ao controle do serviço de inspeção federal (SIFRJ), no período de 1997 a 2003. Arquivo do Instituto Biológico de São Paulo, v. 73, n. 1, p. 83-87, 2006.

PFUETZENREITER, M. R.; ÁVILA-PIRES, F. D. de. Epidemiologia da Teníase/cisticercose por Taenia solium e Taenia saginata. Ciência Rural, v. 30, n. 3, p. 541-548, 2000.

SANTOS, D. V. et al. Análise das principais lesões encontradas nos abatedouros registrados na CISPOA. Informativo técnico, $n^{\circ}$ 04, 2010. Disponível em: <http://www.saa.rs.gov.br/servicos.php?cod=1>. Acessado em: 18 jan. 2012.

SANTOS, L. M. et al. Cisticercose em suínos. Revista científica eletrônica de Medicina Veterinária, v. 6, n. $10,2008$.

SILVA, M. C. Levantamento soroepidemiológico de cisticercose suína na região centro-sul do Estado do Ceará. Fortaleza: UECE, 2004. Dissertação (Mestrado em Ciências Veterinárias), Universidade Estadual do Ceará, 2004.

SOUZA, V. K. de et al. Regiões anatômicas de maior ocorrência de Cysticercus bovis em bovinos submetidos à inspeção federal em matadouro-frigorífico no município de São José dos Pinhais, Paraná, de julho a dezembro de 2000. Revista Brasileira de Parasitologia Veterinária, v. 16, n. 2, p. 92-96, 2007.

THORNTON, H. Compêndio de inspeção de carnes. 5.ed. São Paulo: Fremag LTDA, 1969, 665p. http://cascavel.ufsm.br/revistas/ojs-2.2.2/index.php/reget 